\title{
Simultaneous Quantitative Assessment of Myocardial Perfusion and Function Using Analysis of Color-Encoded Contrast-Enhanced Images
}

\author{
EG Caiani $^{1,2}$, CE Korcarz ${ }^{1}$, KA Collins ${ }^{1}$, RM Lang $^{1}$, V Mor-Avi ${ }^{1}$ \\ ${ }^{1}$ The University of Chicago, Chicago, Illinois, USA, and ${ }^{2}$ Politecnico di Milano, Milan, Italy
}

\begin{abstract}
We hypothesized that analysis of color-encoded, contrast-enhanced, power modulation images could allow simultaneous quantification of myocardial perfusion and regional $L V$ wall motion. We studied 12 anesthetized pigs at baseline, during acute ischemia and subsequent reperfusion, as well as 8 patients post acute myocardial infarction. Color kinesis was used to color-encode endocardial motion during real-time perfusion imaging with high-energy ultrasound pulses. Wall motion was assessed by calculating regional fractional area changes. Translation-free perfusion analysis was performed in automatically identified myocardial regions of interest. Steady-state intensity and initial rate of contrast replenishment were calculated. In all animals, ischemia caused reversible changes in the images and the calculated perfusion and function indices. A significant decrease in pixel intensity (14\%) and contrast replenishment rate (66\%) in LAD-related segments coincided with a decrease in fractional area change (34\%). In patients, respective perfusion and function indices were $61 \%, 51 \%$ and $58 \%$ lower in segments where perfusion defects and/or regional wall motion abnormalities were noted in grey-scale images. Color-encoded, contrast-enhanced power modulation allows simultaneous real-time quantification of myocardial perfusion and regional LV function.
\end{abstract}

\section{Introduction}

The use of echocardiography in the diagnosis of ischemic heart disease is based on subjective, experiencedependent, visual interpretation of left ventricular (LV) wall motion. Although it is agreed that information on myocardial perfusion would be an important adjunct to wall motion, quantification of perfusion with ultrasound contrast has not yet become a standard clinical technique. We have recently described semi-automated, off-line analysis of contrast-enhanced power modulation images for combined quantitative assessment of myocardial perfusion and regional LV function [1]. Regional LV function was evaluated from endocardial borders automatically detected frame-by-frame using depth-dependent thresholding. Myocardial perfusion was assessed using high-energy ultrasound impulses and calculating indices of contrast replenishment in hand-drawn myocardial regions of interest (ROIs) with manual frame-by-frame realignment [1].

More recently, automated border detection from contrast-enhanced power modulation images was implemented to allow the use of color kinesis for real-time color-encoding and quantitative segmental analysis of wall motion [2-3]. In the present study, we used color-encoding not only for analysis of wall motion but also for automated identification of myocardial ROIs and translation-free analysis of myocardial perfusion [4]. We tested this approach in an animal model of acute ischemia, as well as in a group of patients with acute myocardial infarction.

\section{Methods}

\subsection{Imaging}

Power modulation images were obtained using an S3 transducer (SONOS 5500, Philips). Contrast enhancement was achieved by using i.v. infusion of Definity (BristolMyers Squibb). Infusion rates were optimized for dense opacification of the LV cavity without attenuation, and with clearly visualized intramyocardial contrast. After optimizing image quality, gain settings were optimized for endocardial tracking by acoustic quantification software [5] modified to detect the interface between the bright, contrast-enhanced LV cavity and the relatively dark myocardial tissue. Color kinesis was then activated to color-encode LV systolic endocardial motion [6].

\subsection{Animal study}

Experiments were carried out in 12 anesthetized pigs (20-30 kg). EKG, body temperature, noninvasive blood pressure and expiratory gases were monitored. Continuous lidocaine infusion was used to prevent arrhythmias. An intracoronary balloon catheter was introduced via the right femoral artery into the LAD coronary artery. 
Parasternal short-axis view images were continuously acquired over 20 cardiac cycles during infusion of contrast agent Definity. A high-energy ultrasound pulse (mechanical index 1.6) was transmitted to image post-impulse contrast replenishment [7]. Images were acquired under control conditions, 30sec after complete coronary occlusion, and after balloon deflation. To prevent cardiac translation, the ventilator was stopped during image acquisition.

\subsection{Human study}

Eight consecutive patients $(3 \mathrm{M}, 5 \mathrm{~F})$ post acute myocardial infarction were studied within 24 hours post primary coronary revascularization. Images were obtained in the apical four-chamber view during continuous infusion of Definity with high-energy ultrasound pulses. Digital grey-scale images were reviewed by an experienced echocardiographer who classified regional wall motion as normal or abnormal.

\subsection{Image analysis}

Initially, end-systolic color overlays were analyzed to calculate regional fractional area change (RFAC) in six wedge-shaped segments of the LV wall [2]. These data were displayed as stacked color histograms, where each layer represents the area change in percent of regional enddiastolic area (\%REDA) that occurred during the time interval between two consecutive frames.

Myocardial perfusion was quantified using the following automated procedure. For each consecutive end-systolic frame (fig. 1,A), the color-encoded information was used to identify the endocardial border, which was then used to automatically define myocardial ROIs. A binary black and white mask was created wherein value 1 was assigned to all colored pixels and 0 was assigned to all other pixels. For each pixel in the mask image, the sum of differences with its 8 nearest neighbors was calculated, and a parametric gradient image was created by displaying these values. Endocardial border was automatically identified from the gradient image as a continuous closed contour surrounding the LV cavity centroid. The extracted endocardial contour was smoothed using a 5-point window weighted averaging of both $\mathrm{x}$ and $\mathrm{y}$ coordinates. Subsequently, the smooth contour (fig. 1,B) was used to generate a band of fixed width (15 to 20 pixels) around the LV cavity, i.e. in the myocardium (fig. 1,C). This band was then automatically divided into six wedge-shaped segments (fig. 1,D) corresponding to those used to quantify wall motion.

For each segment, mean power modulation pixel intensity after high-energy pulse was calculated for each end-systolic frame and plotted over time, resulting in timeintensity curves reflecting contrast replenishment. Six consecutive end-systolic frames, starting 5 heartbeats after the high-energy pulse, were averaged to represent regional pixel intensity at a steady state during baseline, ischemia and reperfusion phases. The slope of the linear regression of the first 5 beats after the pulse was used as an index of contrast replenishment rate.
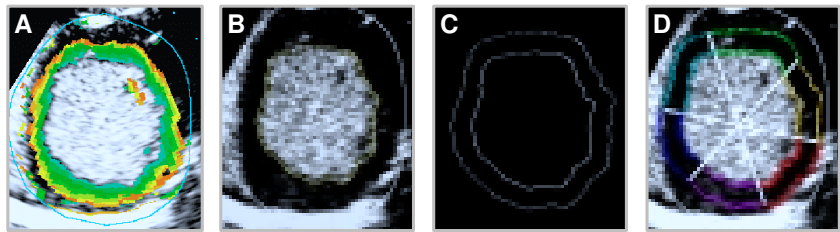

Figure 1. Automated identification of myocardial regions of interest for perfusion analysis from color-encoded, contrastenhanced images (A) was achieved by extracting the endsystolic endocardial border (B), which was used to generate a band of fixed width around the LV cavity (C), and then divided into six wedge-shaped myocardial segments (D).

\subsection{Statistical analysis}

Post-impulse steady-state contrast levels, replenishment rates and fractional area changes, normalized by their control values, were averaged for all animals during baseline, coronary occlusion and reperfusion. Two-way analysis of variance was used to test the differences between phases for all segments. Differences were considered significant for $\mathrm{p}<0.05$, compared to control conditions.

Post-impulse steady-state contrast intensities and replenishment rates were averaged for all patients in each segment. Segments where perfusion defects were noted were compared against those classified as normal. Similarly, fractional area changes were averaged separately for hypokinetic segments and normokinetic segments according to expert interpretation of grey-scale images. Differences between segments were tested using t-test.

\section{Results}

\subsection{Animal study}

In all animals, power modulation imaging during contrast infusion provided uniform opacification of the LV cavity, which allowed automated tracking and color encoding of endocardial motion. These images also allowed simultaneous visualization of intramyocardial contrast. Analysis time ranged between 2 to 5 min per image sequence on a $1.6 \mathrm{MHz}$ personal computer.

Coronary occlusions resulted in visible wall motion abnormalities in the LAD territory, which were reversed during reperfusion. The effects of coronary occlusion and 
reperfusion on the contrast-enhanced power modulation images are shown in figure 2 . In all animals, a decrease in grey-scale pixel intensity in the LAD territory (fig. 2, top middle; arrow), compared to control image (fig. 2, top left), reflected a perfusion defect caused by coronary occlusion. This altered perfusion pattern was accompanied by thinning of the color band (fig. 2, bottom middle; arrow), compared to the control image (fig. 2, bottom left), reflecting ischemia-induced hypokinesis. Both changes in the greyscale image and the color overlay were reversed during reperfusion (fig. 2, right). No concurrent changes were noted outside the LAD territory.

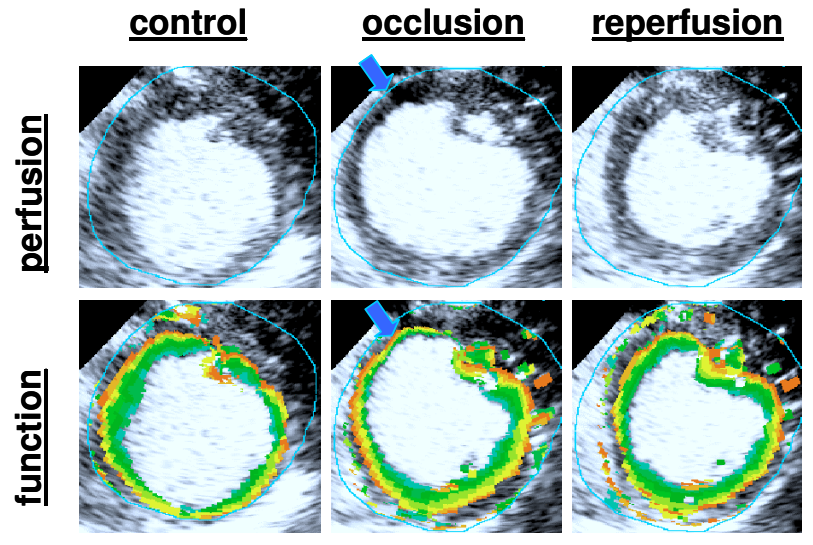

Figure 2. Example of contrast-enhanced power modulation images obtained in a pig with (bottom) and without (top) colorencoding of endocardial motion, during control conditions (left), LAD coronary balloon occlusion (middle), and reperfusion (right).
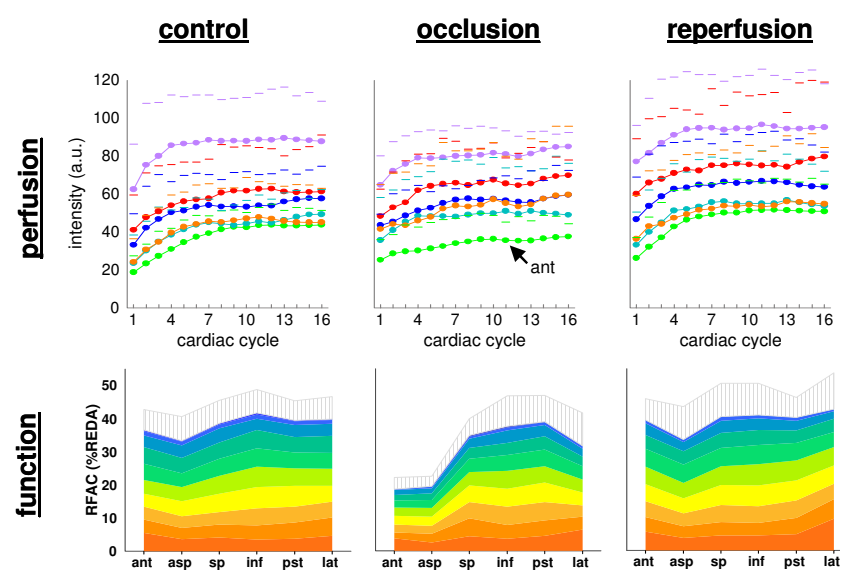

Figure 3. Regional replenishment curves (top) and fractional area change (RFAC) histograms averaged for 12 pigs under control conditions (left), during coronary occlusion (middle) and reperfusion (right). Note ischemia-induced changes in both perfusion and function patterns (see text for details).
These reversible perfusion and function defects in the LAD territory were confirmed by changes in contrast replenishment curves and stacked RFAC histograms. Figure 3 shows these data averaged for all 12 animals, and figure 4 presents the summary of control-normalized perfusion and function indices obtained at the different experimental phases. Ischemia-induced changes in perfusion were evident in the LAD territory, mostly in the anterior segment (fig. 3, top), where perfusion indices decreased significantly during coronary occlusion (fig. 4, left). At the same time, fractional area changes in the anterior and anteroseptal segments decreased significantly compared to control conditions (fig. 3, bottom middle, and fig. 4, right). In these segments, reperfusion hyperemia resulted in higher perfusion indices compared to control, reaching higher steady-state intensities. In contrast, in the non-ischemic segments, neither perfusion nor function indices changed significantly throughout the experiment.

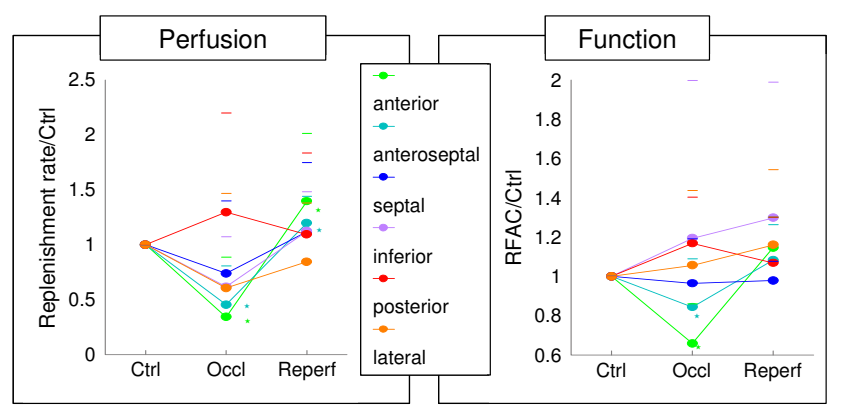

Figure 4. Summary of control-normalized indices of regional myocardial perfusion and LV wall motion. Both perfusion and function indices decreased as a result of coronary occlusion in the area supplied by the occluded artery ( $\star \mathrm{p}<0.05$ vs. control). These ischemic changes were reversed during reperfusion.

\subsection{Human study}

In 6/8 patients, expert interpretation of the grey-scale images identified perfusion defects associated with regional wall motion abnormalities: four patients showed no perfusion (fig. 5), while two had "patchy" myocardial contrast enhancement in the LAD territory. The remaining two patients showed delayed contrast replenishment in the LAD segments, accompanied by preserved wall motion.

Myocardial contrast was difficult to visualize and quantify in the lateral wall. Conversely, in the septum, both quantitative perfusion indices were lower in segments where perfusion defects were noted $(\mathrm{p}<0.05)$. Thin color bands were noted in areas of hypokinesis in all patients who had wall motion abnormalities according to expert interpretation of grey-scale images. Analysis of the color information showed significantly lower regional fractional area change in these segments. 

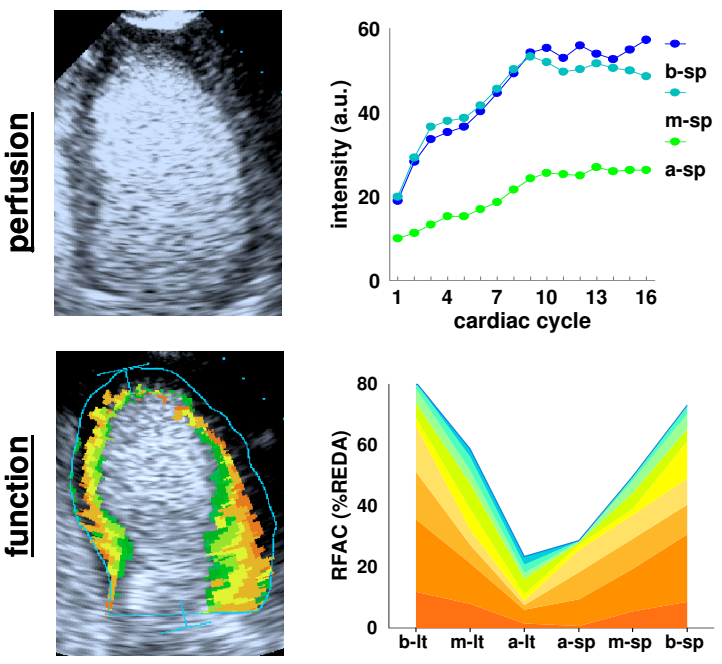

Figure 5. Images obtained in a patient post distal LAD infarction, with (bottom) and without (top) color-encoding. Note the differences between the contrast replenishment curves (top, right) obtained in the apical septal (a-sp) segment where perfusion defect was noted (top, left) and the control mid- and basal-septal (m-sp and b-sp) segments, and the concomitant decrease in fractional area change (RFAC) in this segment (bottom, right) reflecting reduced wall motion (bottom, left).

\section{Discussion}

Echocardiographic diagnosis of coronary heart disease is mainly based on the assessment of LV wall motion. This methodology has been traditionally based on subjective and experience-dependent visual interpretation of dynamic grey-scale images, which remains the widely accepted clinical standard. The notion of extracting information on myocardial perfusion from ultrasound images is highly attractive, and many times has been referred to as the "holy grail" of contrast echocardiography [8]. This goal stimulated multiple technological developments, including new imaging techniques targeted at increased sensitivity for the detection of intramyocardial contrast, such as pulse inversion [9] and power modulation [1].

Recent technological improvements in power modulation imaging included real-time detection and tracking of the endocardial border in contrast-enhanced images. This improvement allowed simultaneous, real-time imaging of intramyocardial contrast and color-encoding of endocardial motion, suitable for quantitative analysis of myocardial perfusion and regional LV function from the same image sequence. We designed our analysis to use the color-encoding information not only for the evaluation of regional wall motion, but also for automated, frame-byframe identification of myocardial ROIs as a basis for translation-free quantification of myocardial perfusion.
The results of our animal protocol showed that myocardial ischemia resulted in noninvasively detectible and quantifiable changes in perfusion and regional LV function. Demonstrating the causal relationship between ischemia and these quantitative indices substantiated the use of this technique in the diagnosis of ischemic heart disease. This approach proved feasible in a small group of patients with acute myocardial infarction by demonstrating the known variety of post-intervention scenarios reflecting variable degrees of success of coronary revascularization and myocardial viability. The availability of this information may prove clinically useful to predict functional recovery in patients post myocardial infarction.

\section{References}

[1] Mor-Avi V, Caiani EG, Collins KA, Korcarz CE, Bednarz JE, Lang RM. Combined assessment of myocardial perfusion and regional left ventricular function by analysis of contrastenhanced power modulation images. Circulation 2001; 104:352-357.

[2] Mor-Avi V, Vignon P, Koch R, Weinert L, Spencer KT, Lang RM. Segmental analysis of Color Kinesis images: New method for quantitative assessment of left ventricular contraction and relaxation. Circulation 1997; 95:2082-2097.

[3] Lang R, Vignon P, Weinert L, Bednarz J, Korcarz C, Sandelski J, Koch R, Prater D, Mor-Avi V. Echocardiographic quantification of regional left ventricular wall motion using Color Kinesis. Circulation 1996; 93:18771885.

[4] Caiani EG, Lang RM, Caslini S, Collins KA, Korcarz CE, Mor-Avi V: Quantification of regional myocardial perfusion using automated, translation-free analysis of contrastenhanced power modulation images. J Am Soc Echocardiogr 2003; 16:116-123.

[5] Bednarz JE, Marcus RH, Lang RM. Technical guidelines for performing automated border detection studies. J Am Soc Echocardiogr 1995; 8:293-305.

[6] Bednarz J, Vignon P, Mor-Avi V, Weinert L, Koch R, Spencer KT, Lang RM. Color kinesis: Principles of operation and technical guidelines. Echocardiography 1998; 15:21-34.

[7] Wei K, Jayaweera AR, Firoozan S, Linka A, Skyba DM, Kaul S. Quantification of myocardial blood flow with ultrasound-induced destruction of microbubbles administered as a constant venous infusion. Circulation 1998; 97:473-483.

[8] Feinstein SB. Myocardial perfusion imaging: contrast echocardiography today and tomorrow [editorial]. J Am Coll Cardiol 1986; 8:251-253.

[9] Meairs S, Daffertshofer M, Neff W, Eschenfelder C, Hennerici M. Pulse-inversion contrast harmonic imaging: ultrasonographic assessment of cerebral perfusion [letter]. Lancet 2000; 355:550-551.

Address for correspondence:

Victor Mor-Avi, University of Chicago, MC 5084,

5841 S. Maryland Ave., Chicago, IL 60637, U.S.A.

E-mail address: moravi@ medicine.bsd.uchicago.edu. 